JPE 11-6-16

\title{
Design of Input Filters Considering the Stability of STATCOM Systems
}

\author{
Guopeng Zhao ${ }^{\dagger}$, Jinjun Liu*, and Minxiao HAN** \\ $\dagger^{* *}$ School of Electrical and Electronic Engineering, North China Electric Power University, Beijing, China \\ * School of Electrical Engineering, Xi' an Jiaotong University, Xi'an, China
}

\begin{abstract}
Previous publications regarding the design and specifications of input filters for STATCOMs usually deal with the input filter only, and seldom pay any attention to the influence of the input filters on the performance of the STATCOM systems. A detailed analysis of the influences of input filters on the stability of STATCOM systems and the corresponding design considerations are presented in this paper. Three types of input filters, L filters, LC filters, and LCL filters, are examined separately. The influences of the parameters of input filters on system stability are investigated through frequency domain methods. With direct current control taken as the major control strategy for the STATCOMs, the different situations when adopting different current detection points are covered in this analysis. A comparison between LC filters and LCL filters is also presented with optimized filter parameters. Based on the analysis, the phase margin, as one of the design considerations for the different types of input filters under different current detection schemes, is discussed. This leads to filter parameters that are different than those of the traditional design. Hardware experimental results verify the validity of the above analysis and design.
\end{abstract}

Key Words: Direct current control, Input filter, L filter, LC filter, LCL filter, STATCOM

\section{INTRODUCTION}

A Static Synchronous Compensator (STATCOM) is one of FACTs (flexible AC transmission system) devices. It can be utilized to regulate the voltage, control the power factor, stabilize the power flow and improve the dynamic performance of power systems [1]-[6]. There is a voltage source converter connected to the grid through an input filter in a STATCOM system. There are three types of input filters which are widely used as low pass filters, L filters, LC filters and LCL filters.

The design of the input filter is important. The input filter is used to filter the harmonic current at the switching frequency. It is also related to the compensated reactive power. Many papers have discussed the choice methods for the parameters of the input filters in STATCOMs. For example, in [7]-[12], the design of the inductor of the input filter must satisfy the ripple current requirement, and, in [13]-[15], the voltage drop across the inductor must be considered to design the inductor of the input filter. In [7], the ripple current should be less than $25 \%$. In [8], the voltage drop across the inductor should be less than $10 \%$. Two methods mentioned in these papers are very useful in the choice of parameters for an inductor, because they present the range of the inductor. However, the exact equation for designing an input filter should be studied.

Manuscript received Nov. 14, 2010; revised Sep. 4, 2011

Recommended for publication by Associate Editor Kyo-Beum Lee.

$\dagger$ Corresponding Author: zhaoguopeng@ncepu.edu.cn

Tel: +86-10-80798443, Fax: +86-10-80798443, North China Electric Power University

* School of Electrical Engineering, Xi'an Jiaotong University, China.
Moreover, the stability of a STATCOM system should be considered as one of aspects in designing an input filter. In practical applications, the filtering performance is usually the main aspect considered in designing an input filter. By using the experience of practical applications and many experimental results, the parameters of the input filter are usually designed. However, if the parameters for the input filters are not suitable for maintaining system stability, parameter adjustment of the controller should be presented. In [16]-[18], investigations of the active damping of input filter resonance are presented.

In this paper, the influences of different types of input filters on STATCOM systems are analyzed in section II. Then, in section III, according to the stability requirements, the corresponding design considerations are presented. Three types of input filters, L filters, LC filters, and LCL filters, are examined separately. A method, based on the phase margin, for designing an input filter is presented. This leads to filter parameters that are different than those of the traditional design. After that, hardware experiments are carried out to verify both the analysis and the specification in section IV. Finally the last section provides conclusions. Based on the analysis and design considerations, the conclusions show that the design of input filters is improved.

\section{EfFect of Different Passive Filters on the STATCOM SYSTEM CHARACTERISTICS}

The direct current control strategy is used in STATCOM systems. A detailed analysis of the influences of input filters on STATCOM systems will be discussed in the follow parts. 


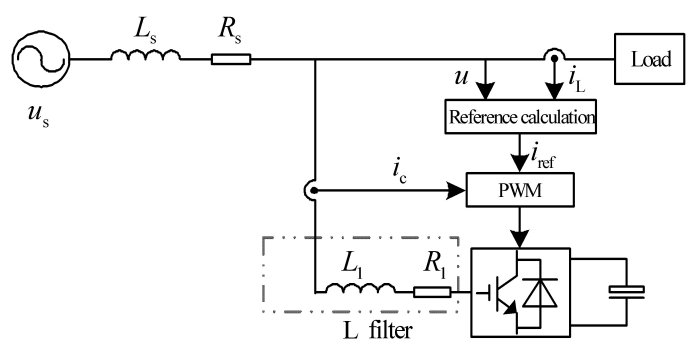

Fig. 1. simplified system configuration.

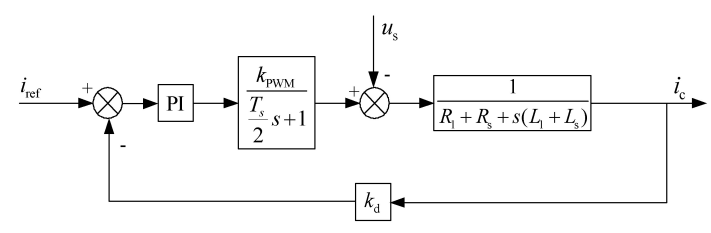

Fig. 2. block diagram of STATCOM.

Also, three types of input filters, L filters, LC filters, and LCL filters, are investigated separately.

In the traditional process of design, when the model of the STATCOM and the parameters are known, then a PI regulator can be used to design a closed loop STATCOM system. However, the parameters for the input filters which are designed by using the traditional methods are not precise enough. In the design process, a change of the input filters is not convenient. Therefore, a common and proper parameter for a PI regulator should be chosen, and the influences of the main circuit parameters on a STATCOM system should be analyzed. Then a more optimized method for the design of input filters is obtained.

\section{A. Analysis of the Influence of the L filter}

A simplified system configuration is shown as Fig.1. A voltage source inverter is used in the main circuit of the STATCOM. The STATCOM is used to compensate the reactive current. $u_{S}$ is the source voltage. $L_{S}$ and $R_{S}$ are the source impedance. $i_{L}$ is the load current. $i_{r e f}$ and $i_{c}$ are the reference current and the output current. $L_{1}$ and $R_{1}$ are the impedances of $\mathrm{L}$ filter. PWM control is carried out. A block diagram of the STATCOM is presented in Fig.2. The PWM part is considered as a proportion and time delay part. $k_{P W M}$ is the proportion. $T_{s}$ is the constant of the delay time of the PWM. $k_{d}$ is the feedback proportion. $k_{p}$ is the value of the proportion. Because the value of the integration is very small in practical applications, the value of the proportion is mainly considered.

The open loop transfer function is shown in (1).

$$
G_{\mathrm{L}_{-} \mathrm{P}}(s)=\frac{k_{\mathrm{d}} k_{\mathrm{p}} k_{\mathrm{PWM}}}{\left(s \frac{T_{\mathrm{s}}}{2}+1\right)\left[R_{1}+R_{\mathrm{s}}+s\left(L_{1}+L_{\mathrm{s}}\right)\right]} .
$$

The cut-off frequencies are $\omega_{1}$ and $\omega_{2}$, where $\omega_{1}<\omega_{2}$. A group of parameters is chosen to analyze the influence of the input filter, where $L_{s}=0.5 \mathrm{mH}, L_{1}=1 \mathrm{mH}, f_{s}=10 \mathrm{kHz}, R_{s}=0.2 \Omega$, $R_{1}=0.1 \Omega, k_{d}=0.06, k_{p}=5, k_{P W M}=80$. Bode plots can be carried out when the parameters $R_{1}$ and $L_{1}$ are changed.

From Fig. 3(a), $R_{1}$ has only a small influence on the magnitude of the low frequency, and has no influence on the magnitude of the medium frequency or the high frequency.

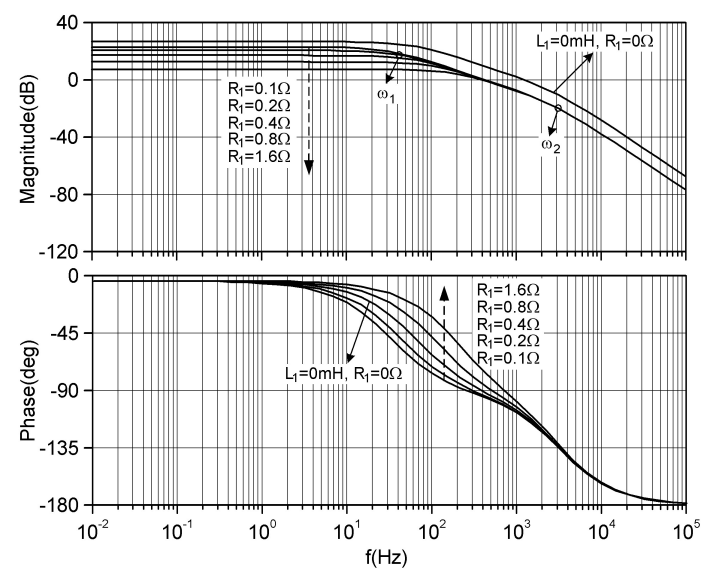

(a) bode plot of changing the variable $R_{1}$.

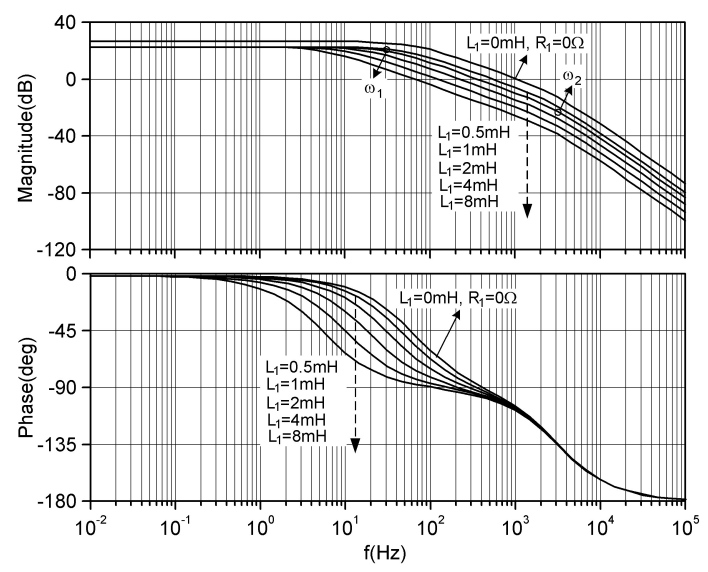

(b) bode plot of changing the variable $L_{1}$.

Fig. 3. bode plots of open loop transfer function.

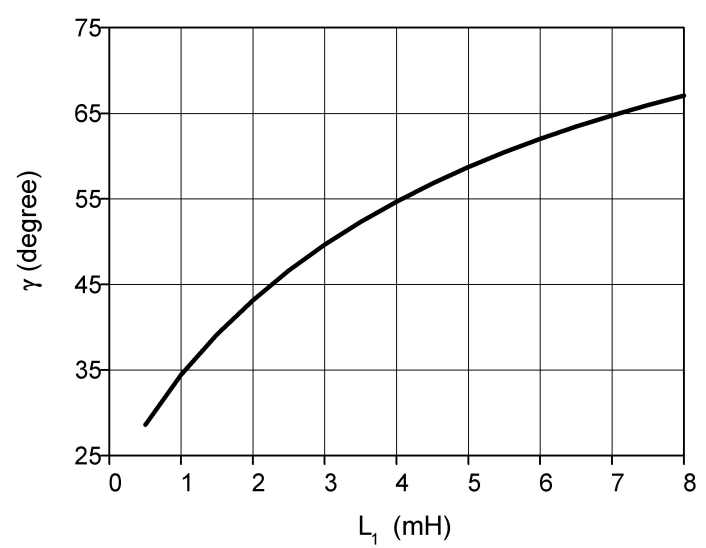

Fig. 4. relationship between the phase margin $\gamma$ and the inductor $L_{1}$.

When the resistor $R_{1}$ increases, the cut-off frequency increases and the magnitude of the low frequency decreases. From Fig. 3 (b), $L_{1}$ affects the magnitude of the medium frequency and the high frequency, but does not affect the magnitude of the low frequency. When the inductor $L_{1}$ increases, the cut-off frequency decreases. Compared with the situation without the L filter, the cut-off frequency decreases and the effect on the noise attenuation becomes better. The relationship between the phase margin $\gamma$ and the inductor $L_{1}$ is illustrated in Fig. 4. The more the inductor increases, the bigger the phase margin is. 


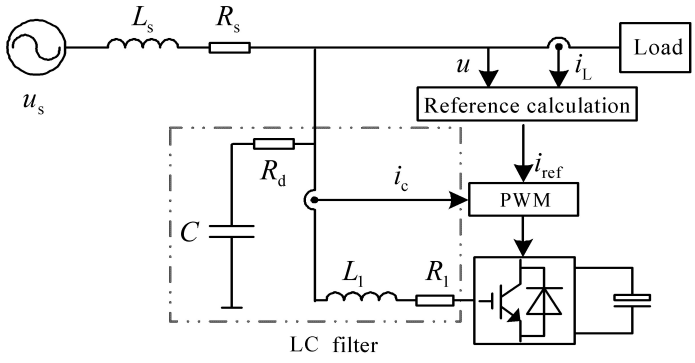

Fig. 5. simplified system configuration.

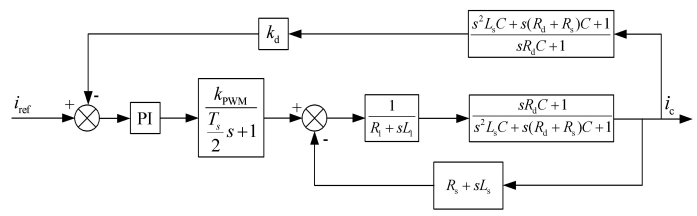

Fig. 6. block diagram of STATCOM.

\section{B. Analysis of the Influence of the LC filter}

For the analysis of the LC filter, two different situations when adopting different current detection points are covered.

1. The feedback loop does not contain a capacitor

The open loop transfer function is shown in (2).

$G_{\mathrm{LC} \_\mathrm{P}}(s)=\frac{k_{\mathrm{d}} k_{\mathrm{p}} k_{\mathrm{PWM}}}{T_{\mathrm{S}} s / 2+1}$

$$
\frac{s^{2} L_{\mathrm{S}} C+s\left(R_{\mathrm{S}}+R_{\mathrm{d}}\right) C+1}{\left(s L_{1}+R_{1}\right)\left[s^{2} L_{\mathrm{S}} C+s\left(R_{\mathrm{S}}+R_{\mathrm{d}}\right) C+1\right]+\left(s R_{\mathrm{d}} C+1\right)\left(s L_{\mathrm{S}}+R_{\mathrm{S}}\right)}
$$

The resonance frequency is shown as (3).

$$
f_{\text {res }}^{\prime}=\frac{1}{\omega_{\text {res }}^{\prime}}=\frac{1}{2 \pi} \sqrt{\frac{L_{\mathrm{s}}+L_{1}}{L_{\mathrm{s}} L_{1} C}}
$$

Fig. 7 shows a Bode plot. The influence of the LC filter on the system characteristics can be seen in Fig. 7. It is nearly the same as the L filter.

From Fig. 7(a), when the inductor $L_{1}$ increases, the cutoff frequency $\omega_{1}$ as well as the magnitude of the medium frequency and the high frequency decrease. Compared with the situation without the LC filter, the cut-off frequency, the resonance frequency of the LC filter $f_{\text {res }}$ and the resonance frequency of the system $f_{\text {res }}$ decrease. Also, the effect on the noise attenuation becomes better. The relationship between the phase margin $\gamma$ and the inductor $L_{1}$ is illustrated in Fig. 8(a). The more the inductor increases, the bigger the phase margin becomes. In Fig. 7(b), when the capacitor $C$ increases, the cut-off frequency increases and the effect on noise attenuation becomes worse. As can be seen in Fig. 8(b), the more the capacitor increases, the bigger the phase margin becomes. $R_{d}$ has only a small influence on the magnitude at the resonance frequency.

2. The feedback loop contains a capacitor

The open loop transfer function is shown in (4).

$$
\begin{aligned}
& G_{\mathrm{LC}_{-} \mathrm{P}}(s)=\frac{k_{\mathrm{d}} k_{\mathrm{p}} k_{\mathrm{PWM}}}{s \frac{T_{\mathrm{s}}}{2}+1} . \\
& \frac{s R_{\mathrm{d}} C+1}{\left(s L_{1}+R_{1}\right)\left[s^{2} L_{\mathrm{S}} C+s\left(R_{\mathrm{s}}+R_{\mathrm{d}}\right) C+1\right]+\left(s R_{\mathrm{d}} C+1\right)\left(s L_{\mathrm{s}}+R_{\mathrm{s}}\right)}
\end{aligned}
$$

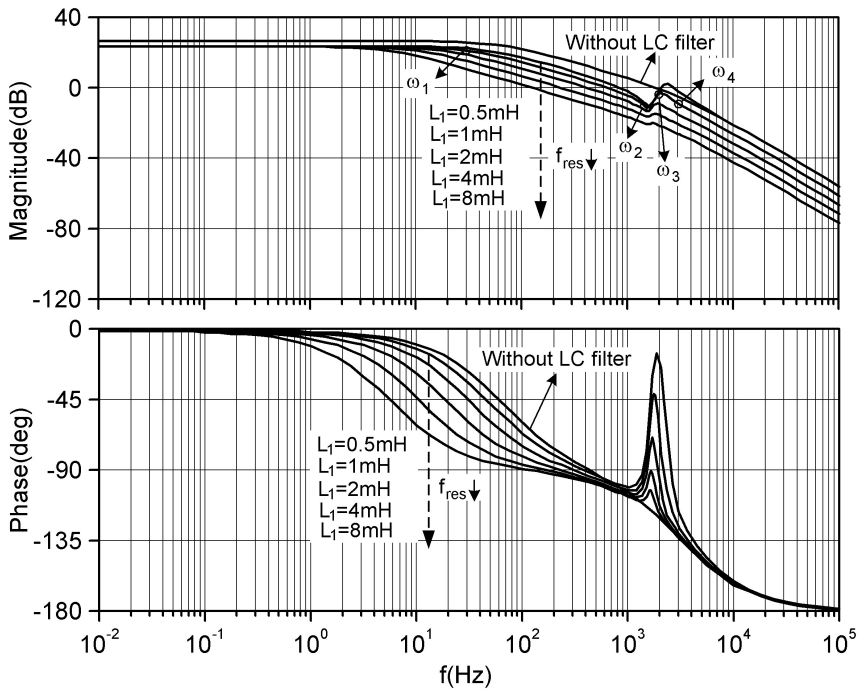

(a) bode plot of changing the variable $L_{1}$.

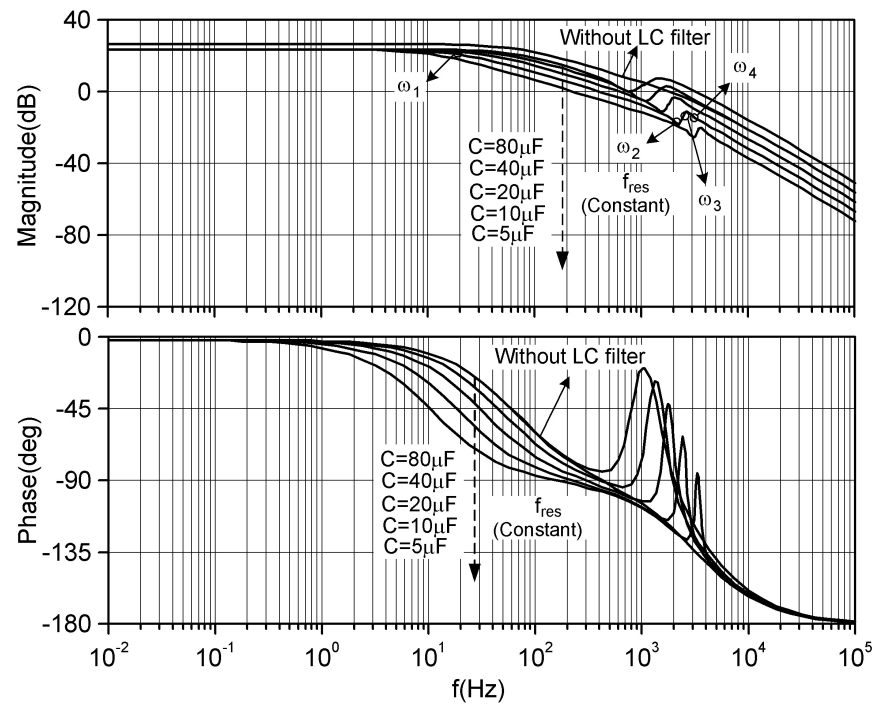

(b) bode plot of changing the variable $C$.
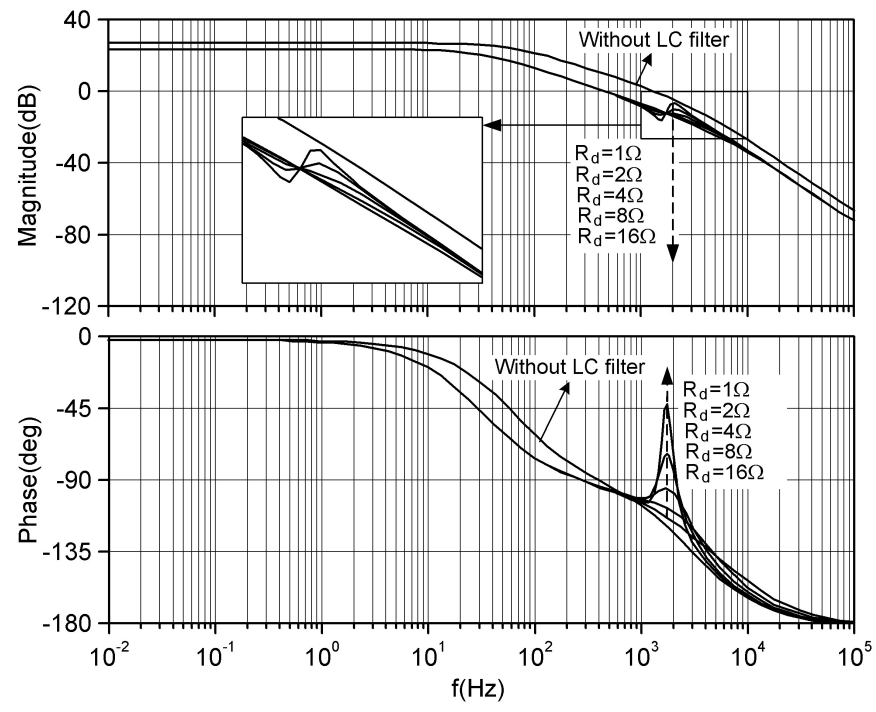

(c) bode plot of changing the variable $R_{d}$.

Fig. 7. bode plots of open loop transfer function. 


$$
\begin{aligned}
& G_{\mathrm{LCL}_{-} \mathrm{P}}(s)=\frac{k_{\mathrm{d}} k_{\mathrm{p}} k_{\mathrm{PWM}}}{s \frac{T_{\mathrm{s}}}{2}+1} \cdot \frac{s^{2}\left(L_{\mathrm{S}}+L_{2}\right) C+s\left(R_{\mathrm{S}}+R_{2}+R_{\mathrm{d}}\right) C+1}{\left(s L_{1}+R_{1}\right)\left[s^{2}\left(L_{\mathrm{S}}+L_{2}\right) C+s\left(R_{\mathrm{S}}+R_{2}+R_{\mathrm{d}}\right) C+1\right]+\left(s R_{\mathrm{d}} C+1\right)\left[s\left(L_{\mathrm{S}}+L_{2}\right)+R_{\mathrm{S}}+R_{2}\right]} \\
& G_{\mathrm{LCL}_{-} \mathrm{P}}(s)=\frac{k_{\mathrm{d}} k_{\mathrm{p}} k_{\mathrm{PWM}}}{s \frac{T_{\mathrm{S}}}{2}+1} \cdot \frac{s R_{\mathrm{d}} C+1}{\left(s L_{1}+R_{1}\right)\left[s^{2}\left(L_{\mathrm{S}}+L_{2}\right) C+s\left(R_{\mathrm{S}}+R_{2}+R_{\mathrm{d}}\right) C+1\right]+\left(s R_{\mathrm{d}} C+1\right)\left[s\left(L_{\mathrm{S}}+L_{2}\right)+R_{\mathrm{S}}+R_{2}\right]}
\end{aligned}
$$

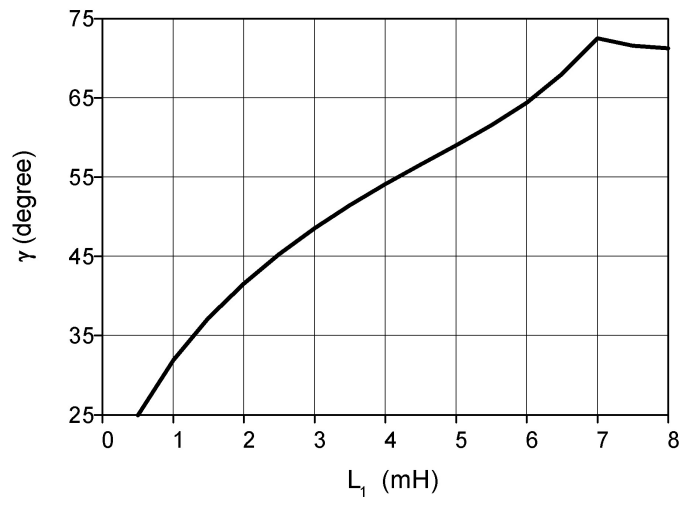

(a) relationship between the phase margin $\gamma$ and the inductor $L_{1}$.

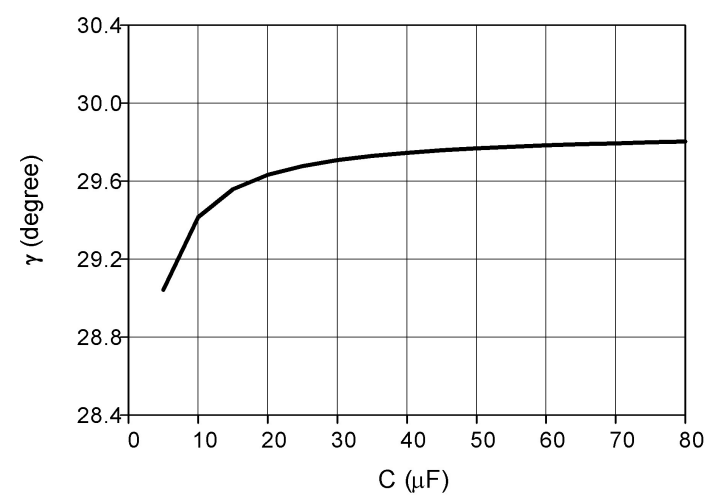

(b) relationship between the phase margin $\gamma$ and the capacitor c.

Fig. 8. relationship between the phase margin $\gamma$ and the variables.

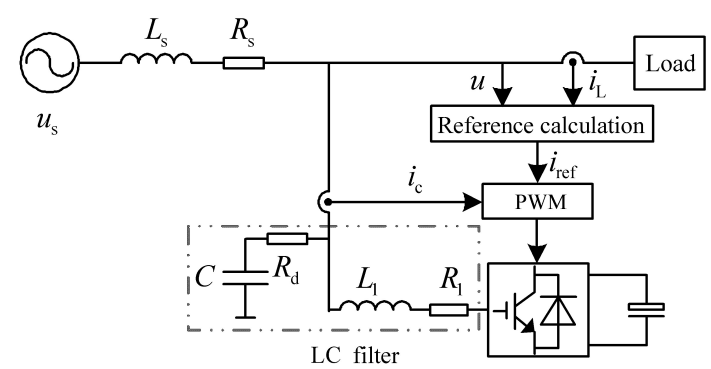

Fig. 9. simplified system configuration.

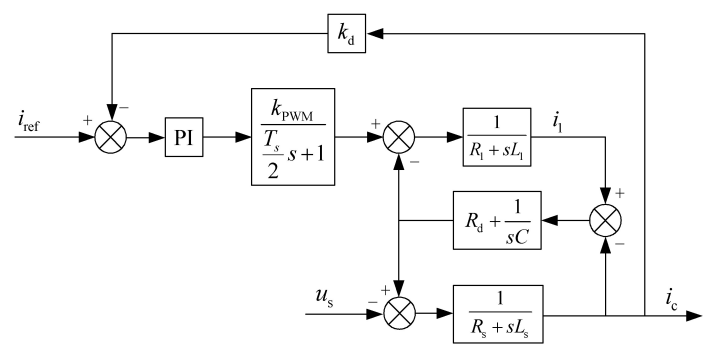

Fig. 10. block diagram of STATCOM.

Fig.11 is a Bode plot. The influence of the LC filter on the system characteristics can be seen in Fig. 11.

The influences of the variables $L_{1}, C$ and $R_{d}$ on the system characteristics are the same as when the feedback loop does not contain a capacitor. The more the inductor $L_{1}$ increases, the bigger the phase margin becomes. However, by increasing the variable $C$, the phase margin decreases, and the system changes from the unstable state to the stable state.

\section{Analysis on the Influence of the LCL filter}

1) Analysis of the Influence of the LCL filter: There are also two different situations when adopting different current detection points in the analysis. A simplified system configuration and a block diagram of the STATCOM can be presented, and an open loop transfer function can be obtained. Then, the analysis can be carried out.

1. The feedback loop does not contain a capacitor

The open loop transfer function is shown in (5).

The influences of $L_{1}, C$ and $R_{d}$ on the STATCOM system are the same as with the LC filter, and the influence of $L_{2}$ is mainly analyzed here.

The inductor $L_{2}$ affects the magnitude of the low frequency and the medium frequency, while the effect on the high frequency noise attenuation does not change. However, the peak value of the resonance may cross the zero value of the magnitude axes several times, and this issue should be paid attention to when a system is designed. The more the inductor $L_{2}$ increases, the bigger the phase margin becomes.

2. The feedback loop contains a capacitor

The open loop transfer function is shown in (6).

The influences of the variables $L_{1}, C$ and $R_{d}$ on the system characteristics are the same as when the feedback loop does not contain a capacitor. The more the inductor $L_{1}$ increases, the bigger the phase margin becomes. However, by increasing the variable $L_{2}$, the phase margin decreases, and the system changes from the unstable state to the stable state. 


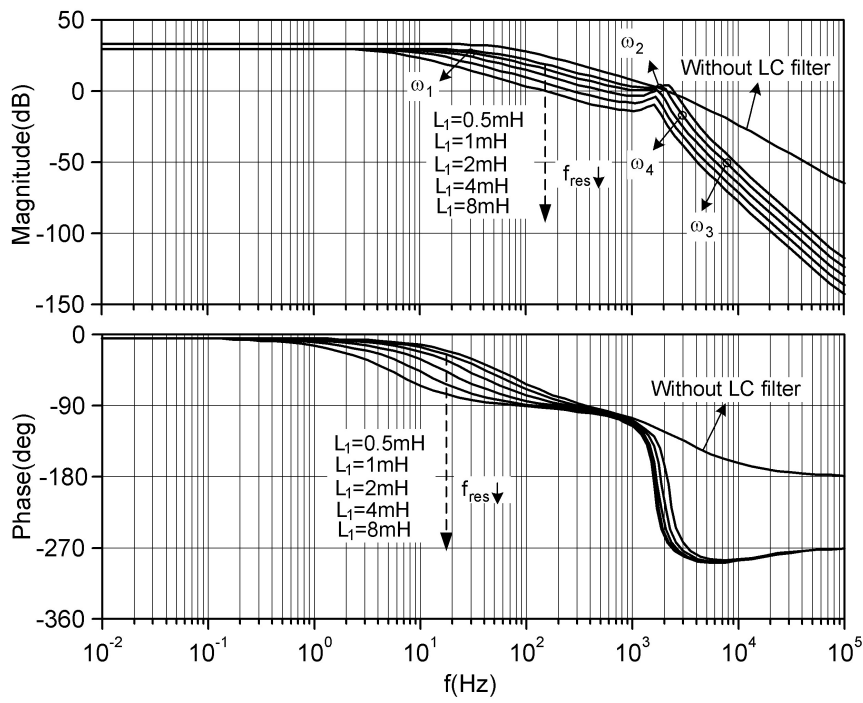

(a) bode plot of changing the variable $L_{1}$.

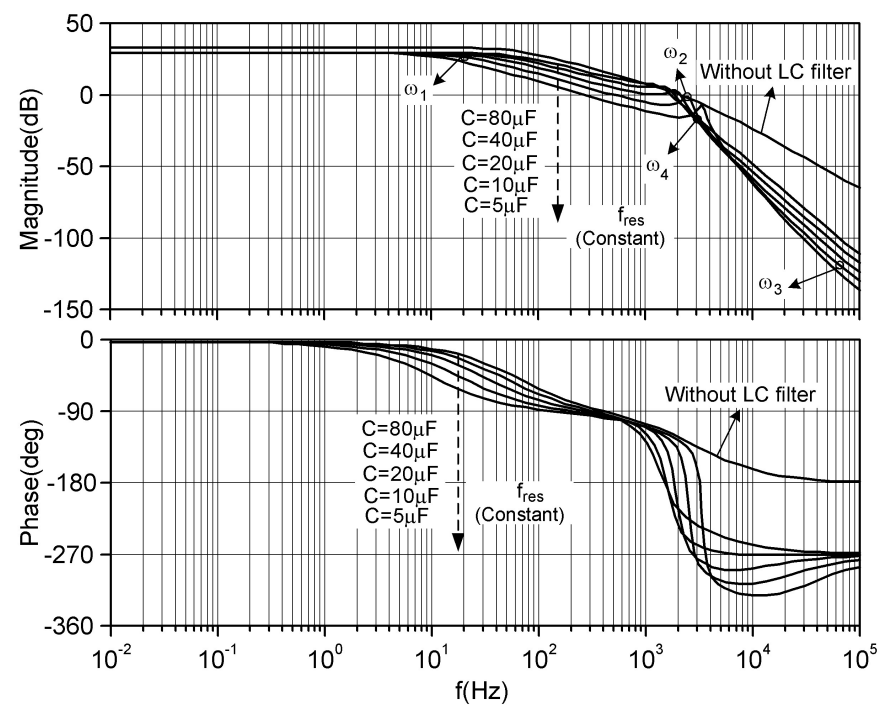

(b) bode plot of changing the variable $C$.
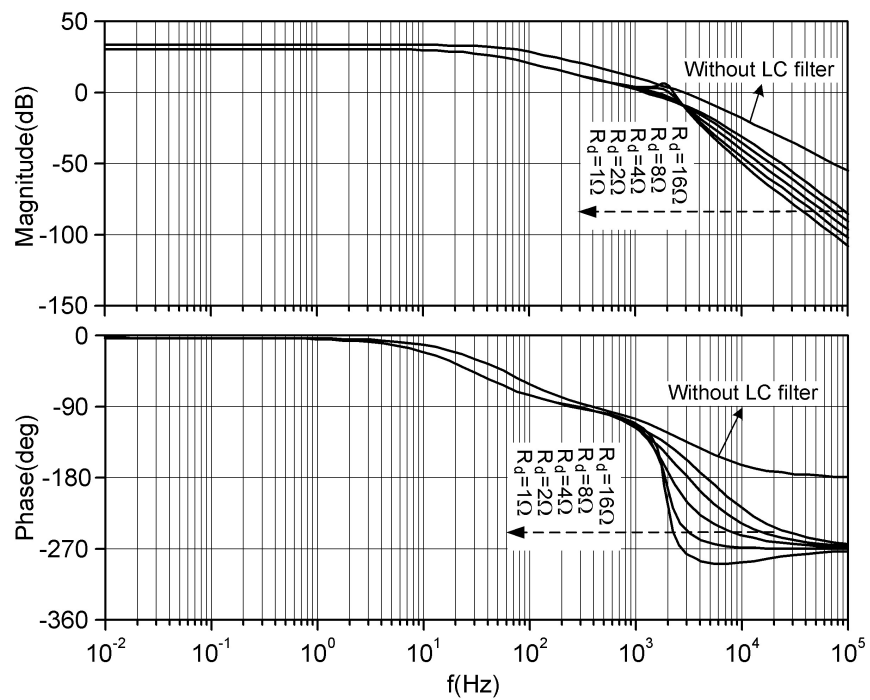

(c) bode plot of changing the variable $R_{d}$.

Fig. 11. bode plots of open loop transfer function.

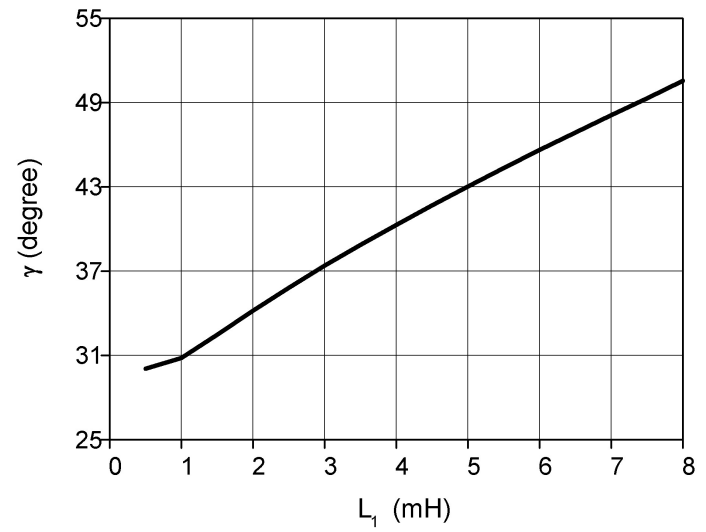

(a) relationship between the phase margin $\gamma$ and the inductor $L_{1}$.

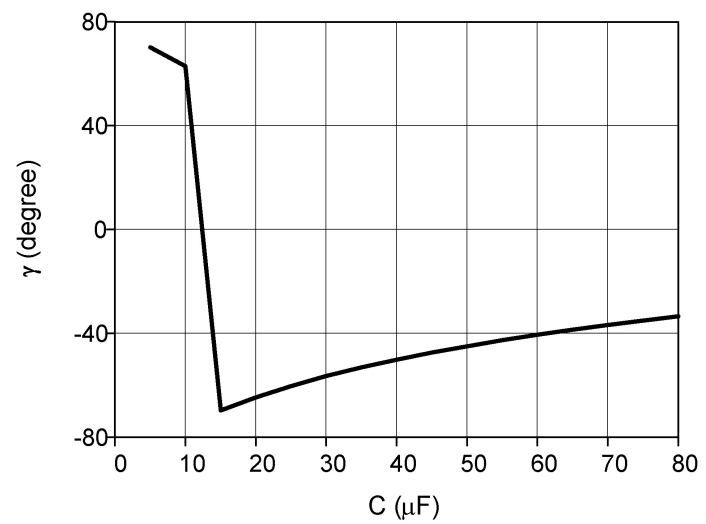

(b) relationship between the phase margin $\gamma$ and the capacitor C.

Fig. 12. relationship between the phase margin $\gamma$ and the variables.

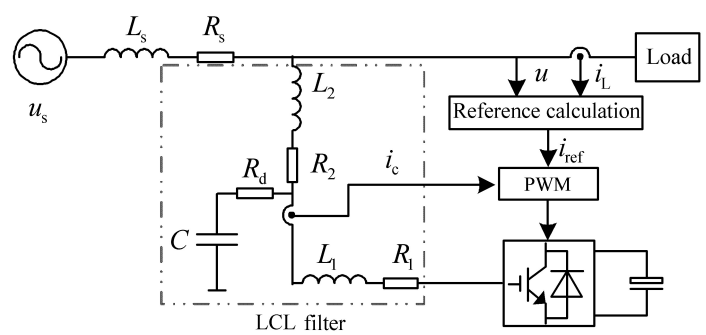

Fig. 13. simplified system configuration.

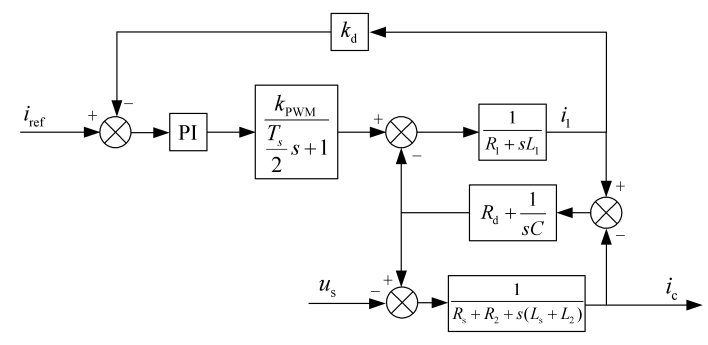

Fig. 14. block diagram of STATCOM. 


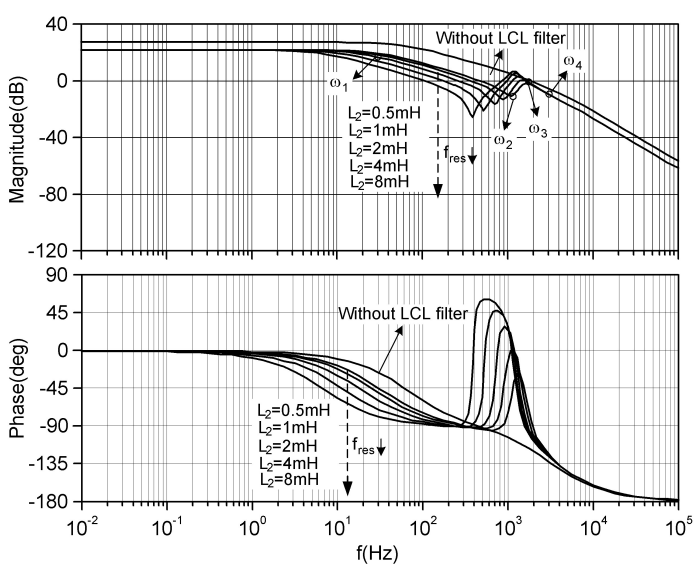

Fig. 15. bode plot of changing the variable $L_{2}$.

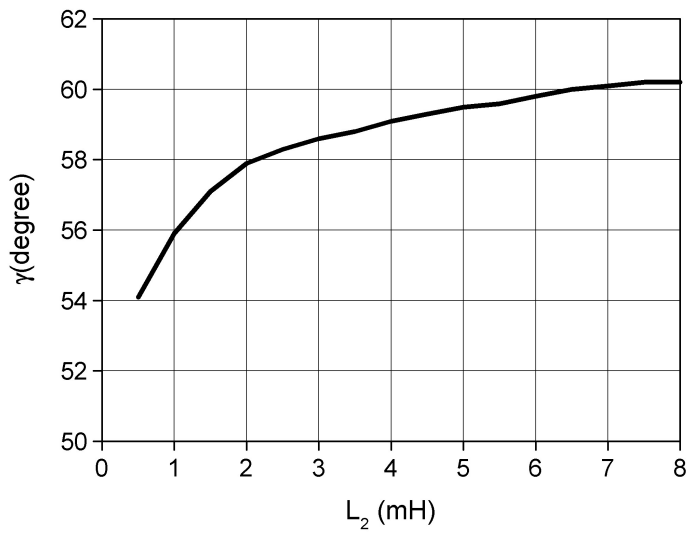

Fig. 16. relationship between the phase margin $\gamma$ and the variable $L_{2}$.

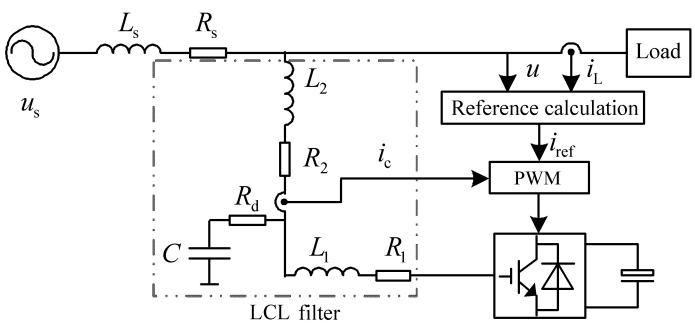

Fig. 17. block diagram of STATCOM.

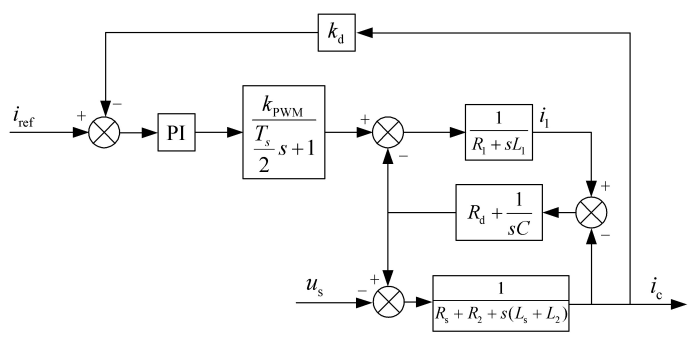

Fig. 18. block diagram of STATCOM.

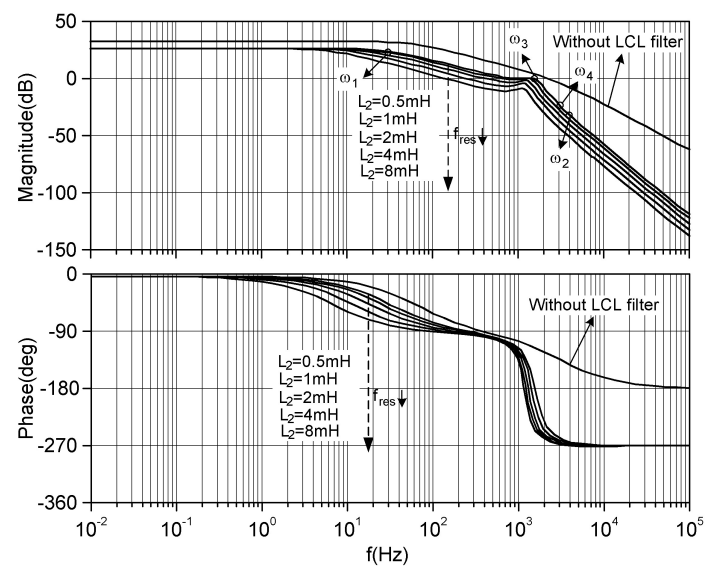

Fig. 19. bode plot of changing the variable $L_{2}$.

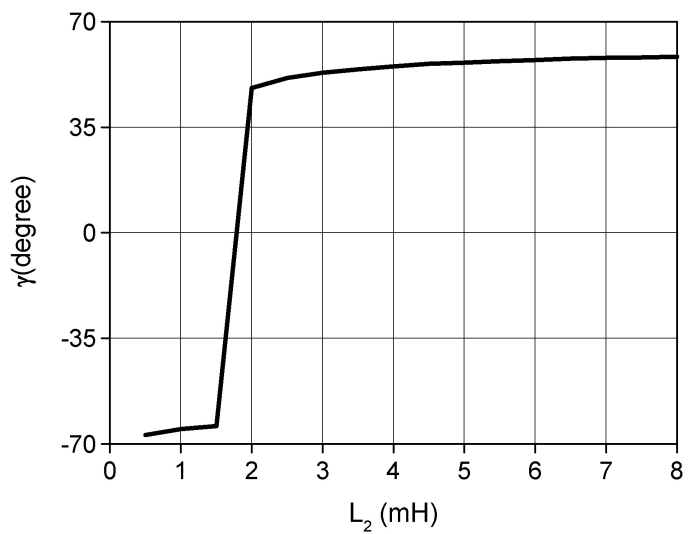

Fig. 20. relationship between the phase margin $\gamma$ and the variable $L_{2}$.

\section{DESIGN OF THE INPUT FILTER}

In [7], the value of the inductor should satisfy (7).

$$
L \geq \frac{U_{\mathrm{dc}}}{8 \eta_{\mathrm{L}} f_{\mathrm{sw}} I_{\mathrm{n}}}
$$

where, $U_{d c}$ is the DC side voltage, $I_{n}$ is the rms value of the output current, $f_{s w}$ is the switching frequency, $\eta_{n}$ is the coefficient of the ripple current, and $\eta_{n}<25 \%$.

In [8], the value of the inductor should satisfy (8).

$$
L \leq \frac{\varepsilon_{\mathrm{L}} U_{\mathrm{s}}}{\omega I_{\mathrm{n}}}
$$

where, $\varepsilon_{L}$ is the coefficient of the voltage drop, and $\varepsilon_{L}<10 \%$.

Therefore, the range of the inductor is shown in (9).

$$
\frac{U_{\mathrm{dc}}}{8 \eta_{\mathrm{L}} f_{\mathrm{sw}} I_{\mathrm{n}}} \leq L \leq \frac{\varepsilon_{\mathrm{L}} U_{\mathrm{s}}}{\omega I_{\mathrm{n}}} .
$$

The relationship in (9) is the traditional method for designing an input filter.

\section{A. Design of the L filter}

According to the analysis in part II of this paper, there is a very important relationship between the phase margin $\gamma$ and the variables of the input filter. As a result, the phase margin $\gamma$, considered as one of input filter' parameters, can be used to design the input filter. It is good for both the stability and reliably of the whole STATCOM system. By using the analysis in part II, the variables $R_{1}$ and $R_{S}$ have a very small influence 
at the cut-off frequency $\omega_{c}$, so they can be ignored. The phase equation and the magnitude equations are shown in (10) and (11).

$$
\begin{gathered}
\omega_{\mathrm{c}}=2 f_{\mathrm{sw}} \operatorname{ctg} \gamma \\
A_{\mathrm{L}_{-} \mathrm{P}}\left(\omega_{\mathrm{c}}\right)=\frac{k_{\mathrm{d}} k_{\mathrm{p}} k_{\mathrm{PWM}}}{\sqrt{\left[1+\left(\frac{\omega_{\mathrm{c}} T_{\mathrm{s}}}{2}\right)^{2}\right] \omega^{2}\left(L_{1}+L_{\mathrm{s}}\right)^{2}}}=1
\end{gathered}
$$

where, $f_{s w}$ is the switching frequency. By combining the phase equation with the magnitude equation, the inductor of the $\mathrm{L}$ filter can be carried out as (12). Equation (12) for designing the $\mathrm{L}$ filter can be used in practical applications.

$$
L_{1}=\frac{k_{\mathrm{d}} k_{\mathrm{p}} k_{\mathrm{PWM}} \sin \gamma}{2 f_{\mathrm{sw}} \operatorname{ctg} \gamma}-L_{\mathrm{s}}
$$

\section{B. Design of the LC filter}

The phase margin $\gamma$, considered as one of parameters, can be used to design the LC filter. When the feedback loop does not contain a capacitor, the variables $R_{1}, R_{d}$ and $R_{s}$ have a very small influence at the cut-off frequency $\omega_{c}$, so they can be ignored. The phase equation and the magnitude equation at the cut-off frequency are shown in (13) and (14).

$$
\begin{gathered}
\omega_{\mathrm{c}}=2 f_{\mathrm{sw}} \operatorname{ctg} \gamma \\
A_{\mathrm{LC} \_} \mathrm{P}\left(\omega_{\mathrm{c}}\right)=\frac{k_{\mathrm{d}} k_{\mathrm{p}} k_{\mathrm{PWM}}}{L_{1} L_{\mathrm{s}} C} \bullet \frac{1-\omega_{\mathrm{c}}^{2} L_{\mathrm{s}} C}{\omega_{\mathrm{c}}\left(\omega_{\mathrm{res}}^{\prime}{ }^{2}-\omega_{\mathrm{c}}^{2}\right) \sqrt{\left[1+\left(\frac{\omega_{\mathrm{c}} T_{\mathrm{s}}}{2}\right)^{2}\right]}}=1
\end{gathered}
$$

where, $\omega_{\text {resi }}^{\prime}$ is the resonance frequency. Equation (14) shows the relationship between the inductor $L$ and the capacitor $C$, and it can be used to design an LC filter.

When the feedback loop contains a capacitor, equation (15) can be obtained by using the same method as when the feedback loop does not contain a capacitor. The equation denotes the relationships between the capacitor and the inductor.

$$
C=\frac{2 f_{\mathrm{sw}} \operatorname{ctg} \gamma\left(L_{1}+L_{\mathrm{s}}\right)-k_{\mathrm{d}} k_{\mathrm{p}} k_{\mathrm{PWM}} \sin \gamma}{8 L_{1} L_{\mathrm{s}} f_{\mathrm{sw}}^{3} \operatorname{ctg}^{3} \gamma}
$$

\section{Design of the LCL filter}

The design of the LCL filter is similar to the design of the LC filter. The method can be obtained by using $L_{2}+L_{s}$ to replace $L_{s}$. If the feedback loop does not contain a capacitor, the effect on the high frequency noise attenuation is strongest when $L_{2}=0$. Then, the LCL filter becomes an LC filter. If the feedback loop contains a capacitor, the values of the inductors $L_{1}$ and $L_{2}$ are discussed here when the total value of the two inductors is a constant.

The resonant frequency is $f_{\text {res }}=\sqrt{1 / L_{1} C+1 / L_{2} C} /(2 \pi)$, where $L_{1}+L_{2}=L$. If $L$ is a constant, then $f_{\text {res }}$ is minimal, when $L_{1}=L_{2}=L / 2$. The smaller $f_{\text {res }}$ is, the better the effect on the noise attenuation becomes. Therefore, if $L_{\mathrm{s}}, C$ and $f_{\text {res }}^{\prime}$ are constants, the LCL filter has a smaller total inductor value than the LC filter. As a result, the LCL filter is better than the LC filter.

A comparison of the three types of the input filters, L filters, LC filters, and LCL filters, was carried out and is shown in Fig. 21. The total value of the inductor is $1 \mathrm{mH}$. From Fig. 21,

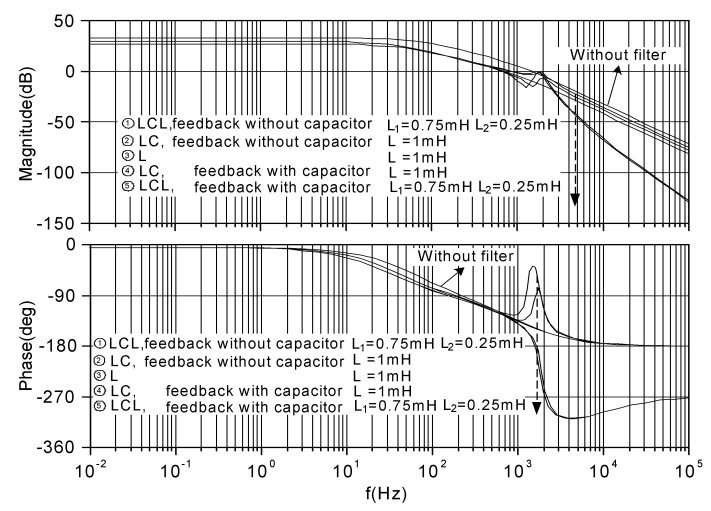

Fig. 21. bode plot of open loop transfer function with different input filter.

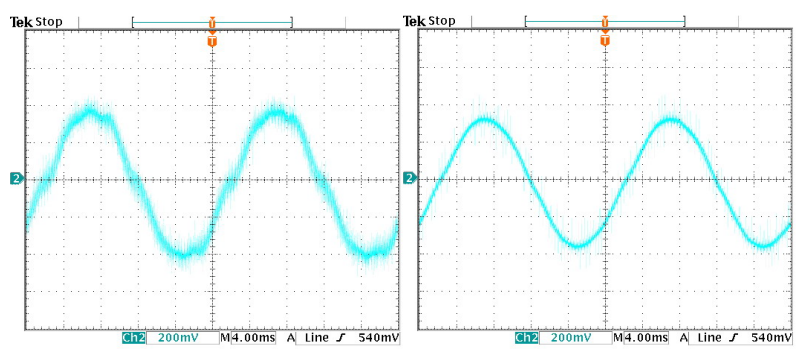

(a) $L_{1}=1 \mathrm{mH}$

(b) $L_{1}=3.5 \mathrm{mH}$.

Fig. 22. output current of STATCOM with L filter ( $t: 4 \mathrm{~ms} / \mathrm{div} i: 2 \mathrm{~A} / \mathrm{div})$.

if the capacitor $C$ is a constant, the LCL filter needs a smaller value for the inductor than an LC filter with the same filtering performance.

\section{EXPERIMENTAL RESULTS}

Different parameters for the input filters have different system characteristics. In order to verify the analysis of the influences on a STATCOM system, hardware experimental results are presented. An experimental system with a low voltage and rating was used to verify the conclusions by using qualitative experimental results.

\section{A. L filter}

From Fig.22, it can be seen that when the inductor $L_{1}$ increased, the values of the medium frequency and the high frequency current decreased. The cut-off frequency of the STATCOM became lower and the filtering performance became better. Moreover, with an increase in $L_{1}$, the current of the low frequency oscillation became smaller and the waveform became more sinusoidal. Then, the system was more stable. The hardware experimental results shown in Fig. 22 verify the validity of the analysis in part II.

\section{B. LC filter}

1. The feedback loop does not contain a capacitor

In Fig.23, when the inductor $L_{1}$ increased, the value of the medium frequency and the high frequency current decreased. It had the same conclusions as L filter. Moreover, when the capacitor $C$ increased, the magnitude of the high frequency current became smaller and the phase margin became bigger. 


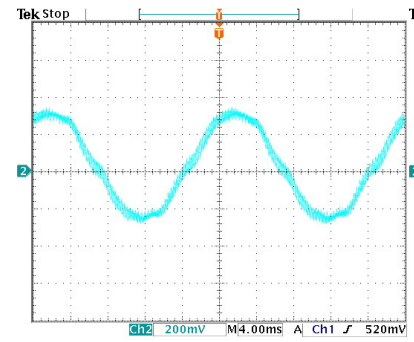

(a) $L_{1}=1 \mathrm{mH}, C=20 \mathrm{mF}$.

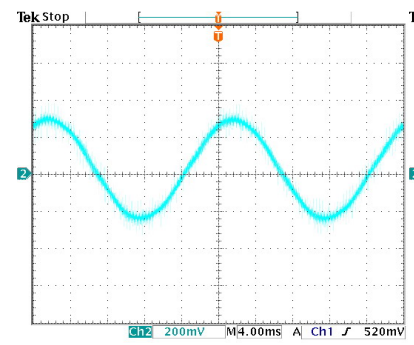

(c) $L_{1}=3.5 \mathrm{mH}, C=50 \mathrm{mF}$.

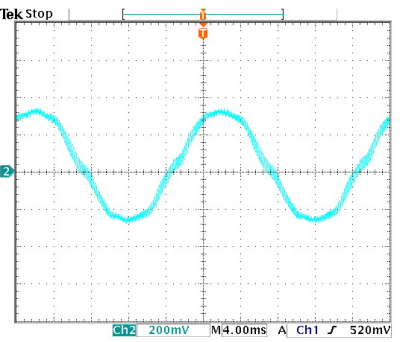

(b) $L_{1}=1 \mathrm{mH}, C=50 \mathrm{mF}$.

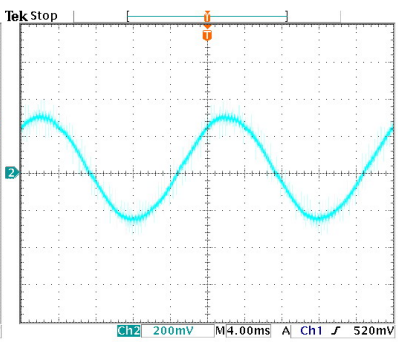

(d) $L_{1}=3.5 \mathrm{mH}, C=80 \mathrm{mF}$.
Fig. 23. output current of STATCOM with LC filter when the feedback loop did not contain capacitor $(t: 4 \mathrm{~ms} / \mathrm{div} i: 2 \mathrm{~A} / \mathrm{div})$.

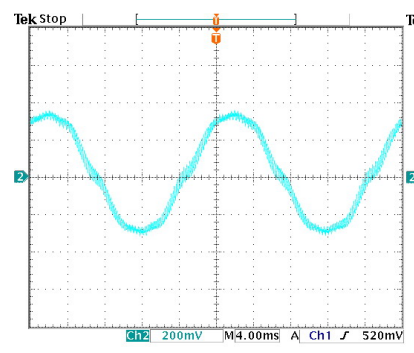

(a) $L_{1}=1 \mathrm{mH}, C=20 \mathrm{mF}$.

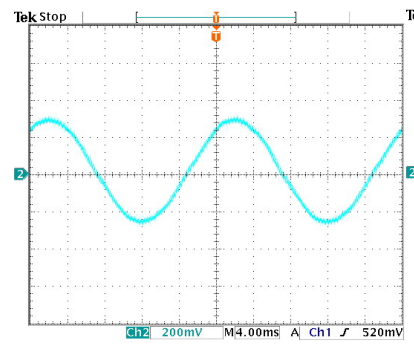

(c) $L_{1}=3.5 \mathrm{mH}, C=50 \mathrm{mF}$.

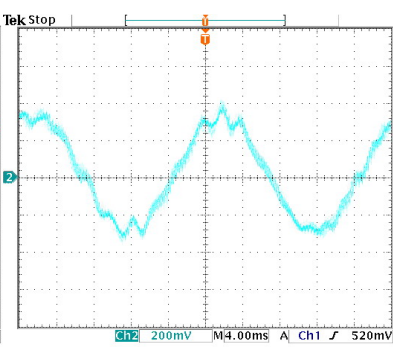

(b) $L_{1}=1 \mathrm{mH}, C=50 \mathrm{mF}$.

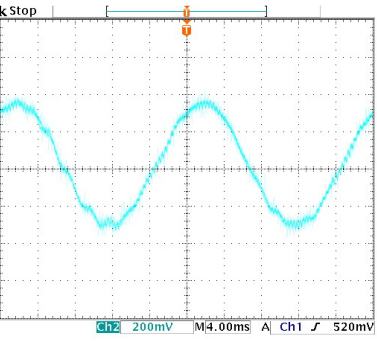

(d) $L_{1}=3.5 \mathrm{mH}, C=80 \mathrm{mF}$.
Fig. 24. output current of STATCOM with LC filter when the feedback loop contained capacitor ( $t$ : $4 \mathrm{~ms} / \operatorname{div} i: 2 \mathrm{~A} / \mathrm{div})$.

The experimental results shown in Fig. 23 verify the analysis above.

2. The feedback loop contains a capacitor

In Fig.24, when the inductor $L_{1}$ increased, the value of the medium frequency and the high frequency current decreased. Moreover, when the capacitor $C$ increased, there was a low frequency oscillation. This was due to a decrease in the phase margin. When the inductor $L_{1}$ increased again, the low frequency oscillation disappeared. This happened because the phase margin increased again when the inductor $L_{1}$ increased again. The experimental results shown in Fig. 24 verify the analysis above.
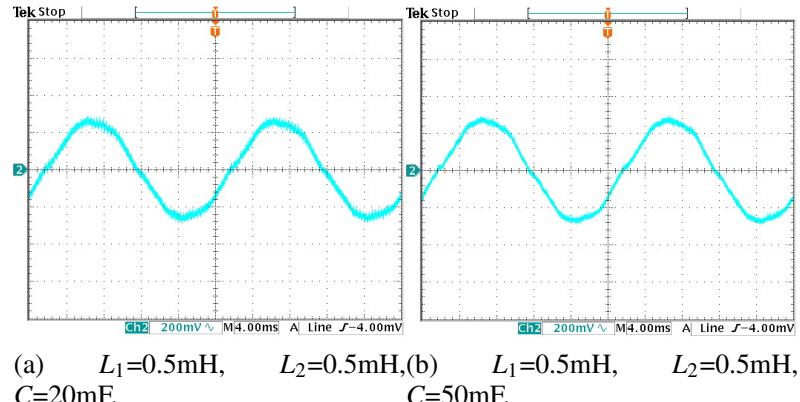

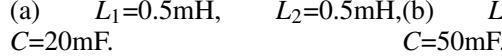

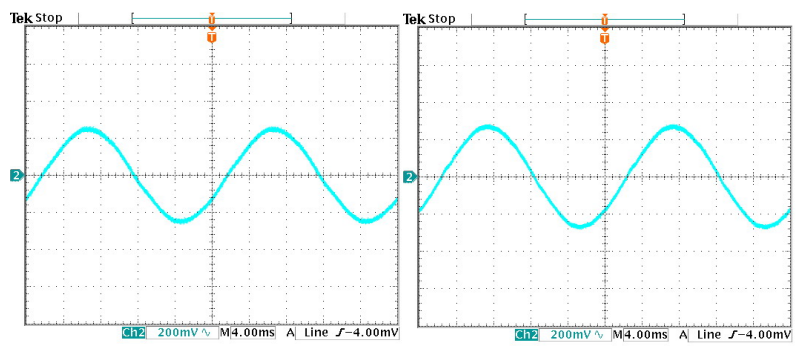

(c)

(d)

$L_{1}=3.5 \mathrm{mH}, L_{2}=0.5 \mathrm{mH}, C=20 \mathrm{mF} . \quad L_{1}=3.5 \mathrm{mH}, L_{2}=0.5 \mathrm{mH}, C=80 \mathrm{mF}$.

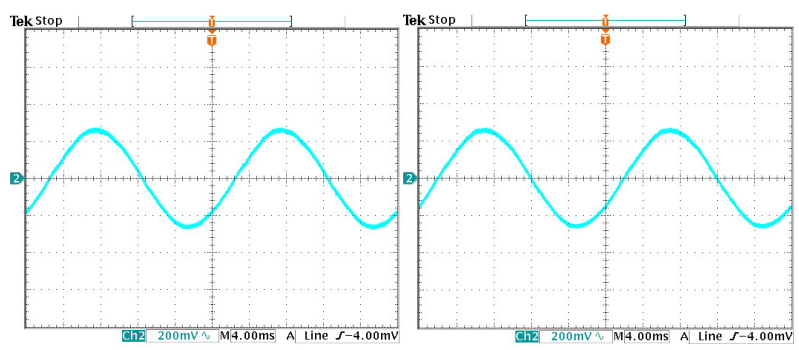

(e) $L_{1}=3.5 \mathrm{mH}, L_{2}=1 \mathrm{mH}, C=20 \mathrm{mF}$.(f) $L_{1}=3.5 \mathrm{mH}, L_{2}=1 \mathrm{mH}, C=80 \mathrm{mF}$.

Fig. 25. output current of STATCOM with LCL filter when the feedback loop did not contain capacitor ( $t: 4 \mathrm{~ms} / \mathrm{div} i: 2 \mathrm{~A} / \mathrm{div})$.

\section{LCL filter}

1. The feedback loop does not contain the capacitor

When the inductors $L_{1}$ and $L_{2}$ increased, the value of the medium frequency and the high frequency current decreased. Moreover, when the capacitor $C$ increased, there was no low frequency oscillation. The system was stable. The experimental results verified the analysis in part II.

\section{The feedback loop contains a capacitor}

When the capacitor $C$ increased, there was a low frequency oscillation. This was due to a decrease in the phase margin. When the inductor $L_{1}$ increased again, the low frequency oscillation disappeared. This happened because the phase margin increased again when the inductor $L_{1}$ increased again. The experimental results verified the analysis in part II.

All of the hardware experimental results verify the validity of the analysis and the design in part II.

\section{CONCLUSIONS}

In this paper, a detailed analysis of the influence of input filters on STATCOM systems is presented, and a new method for designing the input filter of a STATCOM is proposed. The following conclusions can be obtained. 


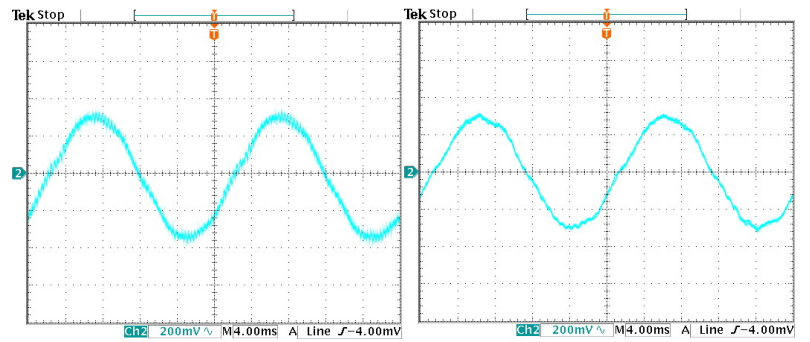

(a) (b) $L_{1}=0.5 \mathrm{mH}, L_{2}=0.5 \mathrm{mH}, C=20 \mathrm{mF} . \quad L_{1}=0.5 \mathrm{mH}, L_{2}=0.5 \mathrm{mH}, C=50 \mathrm{mF}$.

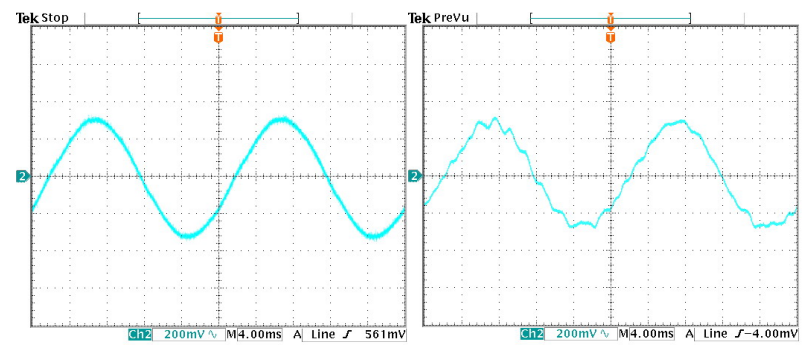

(c) (d)

$L_{1}=3.5 \mathrm{mH}, L_{2}=0.5 \mathrm{mH}, \mathrm{C}=20 \mathrm{mF} . \quad L_{1}=3.5 \mathrm{mH}, L_{2}=0.5 \mathrm{mH}, C=80 \mathrm{mF}$.

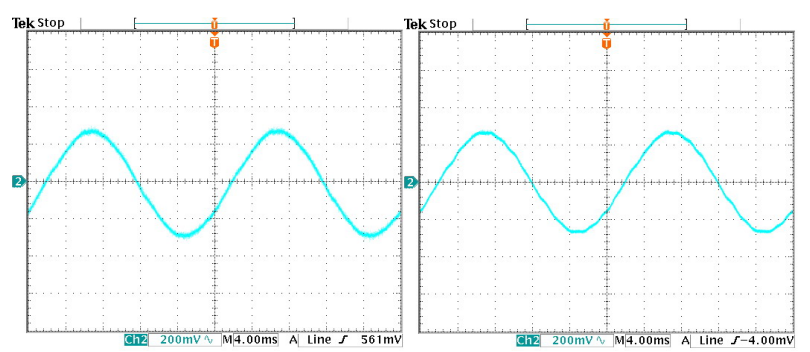

(e) $L_{1}=3.5 \mathrm{mH}, L_{2}=1 \mathrm{mH}, C=20 \mathrm{mF}$. (f) $L_{1}=3.5 \mathrm{mH}, L_{2}=1 \mathrm{mH}, C=80 \mathrm{mF}$.

Fig. 26. output current of STATCOM with LCL filter when the feedback loop contained capacitor $(t: 4 \mathrm{~ms} / \mathrm{div} i: 2 \mathrm{~A} / \mathrm{div})$.

1) For an L filter, when the inductor increases, the phase margin of the system increases.

2) For an LC filter, when the feedback loop does not contain a capacitor, the phase margin of the system increases when the inductor and the capacitor increase. When the feedback loop contains a capacitor, the phase margin of the system increases when the inductor increases.

3) For an LCL filter, when the inductor $L_{2}$ increases, the phase margin of the system increases. The LCL filter needs a smaller total value for the inductor than an LC filter with the same filtering performance.

4) The phase margin can be used to design input filters. The exact equations for designing input filters are proposed.

Based on the analysis and design considerations, the conclusions show that the proposed design of the input filters is more optimal than the traditional method.

\section{REFERENCES}

[1] R. Gupta, A. Ghosh, and A. Joshi, "Performance comparison of vscbased shunt and series compensators used for load voltage control in distribution systems," IEEE Trans. Power Del., Vol. 26, No. 1, pp. 268278, Jan. 2011.

[2] H. M. Pirouz and M. T. Bina, "Modular multilevel converter based statcom topology suitable for medium-voltage unbalanced systems," Journal of Power Electronics, Vol. 10, No. 5, pp. 572-578, Sep. 2010.
[3] C.-K. Kim, V. Sood, and S.-J. Lee, "Dynamic coordination strategies between hvdc and statcom," Journal of Power Electronics, Vol. 9, No. 6, pp. 892-902, Sep., 2009.

[4] B. Singh, P. Jayaprakash, and D. P. Kothari, "A three-phase four-wire dstatcom for power quality improvement," Journal of Power Electronics, Vol. 8, No. 3, pp. 259-267, Jul. 2008.

[5] B. Singh and J. Solanki, "An improved control approach for dstatcom with distorted and unbalanced ac mains," Journal of Power Electronics, Vol. 8, No. 2, pp. 131-140, Apr. 2008.

[6] B. Singh and R. Saha, "Modeling of 18-pulse statcom for power system applications", Journal of Power Electronics, Vol. 7, No. 2, pp. 146-158, Apr. 2007.

[7] T. C. Y. Wang, Z. Ye, G. Sinha, and X. Yuan, "Output filter design for a grid-interconnected three-phase inverter," in Proceeding of IEEE 34th Annual Power Electronics Specialist Conf., Vol. 2, pp. 779-784, 2003.

[8] M. Liserre, F. Blaabjerg, and S. Hansen, "Design and control of an LCLfilter-based three-phase active rectifier," IEEE Trans. Ind. Appl., Vol. 41, No 5, pp.1281-1291, Sep./-Oct. 2005.

[9] Y. Lang, D. Xu, S. R. Hadianamrei, and H. Ma, "A Novel design method of lcl type utility interface for three-phase voltage source rectifier", in Proceeding of IEEE 36th Power Electronics Specialists Conf., pp. 313 $-317,2005$.

[10] T. M. L. de Assis, E. H. Watanabe, L. A. S. Pilotto, and R. B. Sollero, "A new technique to control reactive power oscillations using statcom," in Proceeding of 2002 Harmonics and Quality of Power Conf., pp. 607613, 2002.

[11] P. T. Cheng, S. Bhattacharya, and D. Divan, "Experimental verification of dominant harmonic active filter for high power applications," IEEE Trans. Ind. Appl., Vol. 36, No. 2, pp. 567-577, May/Apr. 2000.

[12] J. Kim, J. Choi, and H. Hong, "Output lc filter design of voltage source inverter considering the performance of controller", in Proceeding of International Conference on Power System Technology, pp. 1659-1664, 2000.

[13] A. K. Guru, J. C. Balda, K. Carr, and Y. Q. Xiang, "Design of a switching-ripple filter for a shunt-connected active power filter," in Proceeding of IEEE Industry Applications Society Annual Meeting, pp. 1364-1368, 1998.

[14] P. A. Dahono, A. Purwadi and Qamaruzzaman, "An LC filter design method for single-phase PWM inverters," in Proceeding of International Conference on Power Electronics and Drive Systems, pp. 571-576, 2124, 1995.

[15] V. Blasko and V. Kaura, "A novel control to actively damp resonance in input LC filter of a three-phase voltage source converter," IEEE Trans. Ind. Appl., Vol.33, No. 2, pp. 542-550, March-April 1997.

[16] T. Erika and D. G. Holmes, "Grid current regulation of a three-phase voltage source inverter with an LCL input filter," IEEE Trans. Power Electron., Vol. 18, No. 3, pp.888-895, May 2003.

[17] C. Wessels, J. Dannehl, and F. W. Fuchs, "Active damping of lcl-filter resonance based on virtual resistor for pwm rectifiers-stability analysis with different filter parameters," in Proceeding of 2008 IEEE Power Electronics Specialists Conf., Rhodes, Greece, pp. 3532-3538, 2008.

[18] M. Routimo and H. Tuusa, "LCL type supply filter for active power filter-comparison of an active and a passive method for resonance damping," in Proceeding of 2007 IEEE Power Electronics Specialists Conf., pp. 2939-2945, 2007.

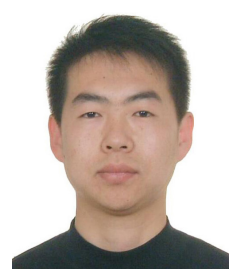

Guopeng Zhao was born in Inner Mongolia Province, China, in 1980. He received his B.S. from the Northwestern Polytechnical University, China, in 2003. He received his M.S. and Ph.D. from Xi' an Jiaotong University (XJTU), China, in 2006 and 2010, respectively, both in Electrical Engineering. He is currently a Lecturer at the North China Electric Power University. His current research interests include power quality control and the application of power electronics in power systems.

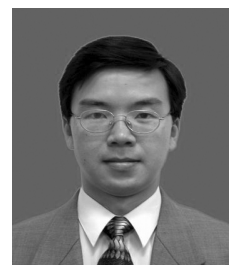

Jinjun Liu was born in Hunan Province, China, in 1970. He received his B.S. and Ph.D. from Xi'an Jiaotong University (XJTU), China, in 1992 and 1997, respectively. In 1998, he led the founding of the XJTU/Rockwell Automation Laboratory. From December 1999 until February 2002, he was with the Center for Power Electronics Systems at Virginia Polytechnic Institute and State University, USA, as a Postdoctoral Research Scholar. He then came back to XJTU and in August of 2002 was promoted to Full Professor and Head of the Power 
Electronics and Renewable Energy Center at XJTU. Now he is also serving as an Associate Dean of the School of Electrical Engineering at XJTU. He has coauthored 3 books, published over 100 technical papers, and received several provincial or ministerial awards for scientific or career achievements. He also received the 2006 Delta Scholar Award. His current research interests include power quality control, renewable energy generation, utility applications of power electronics, and modeling and control of power electronic systems. Dr. Liu has served as the IEEE Power Electronics Society Region 10 Liaison for 3 years. He has been actively involved in the organization of several power electronic international conferences, including the PESC, APEC, and IPEC in Japan, the ICPE in South Korea, and the IPEMC in China, where he served as a committee member, a co-chair, or a session chair.

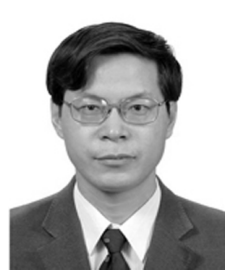

Minxiao HAN was born in Shaanxi, China, in 1963. He received his B.S. from Xi'an Jiaotong University, in 1984, and his M.S. and Ph.D. from the North China Electric Power University (NCEPU), in 1987 and 1995 , respectively. He was a Visiting Research Fellow at the Queen's University of Belfast, U.K. and a Postdoctoral Research Fellow at Kobe University, Japan. He is active in professional society activities and international cooperation in the field of power electronics. His current research interests include the application of power electronics in power systems including HVDC \& FACTS, power quality and the integration of renewable generation systems into power networks. 